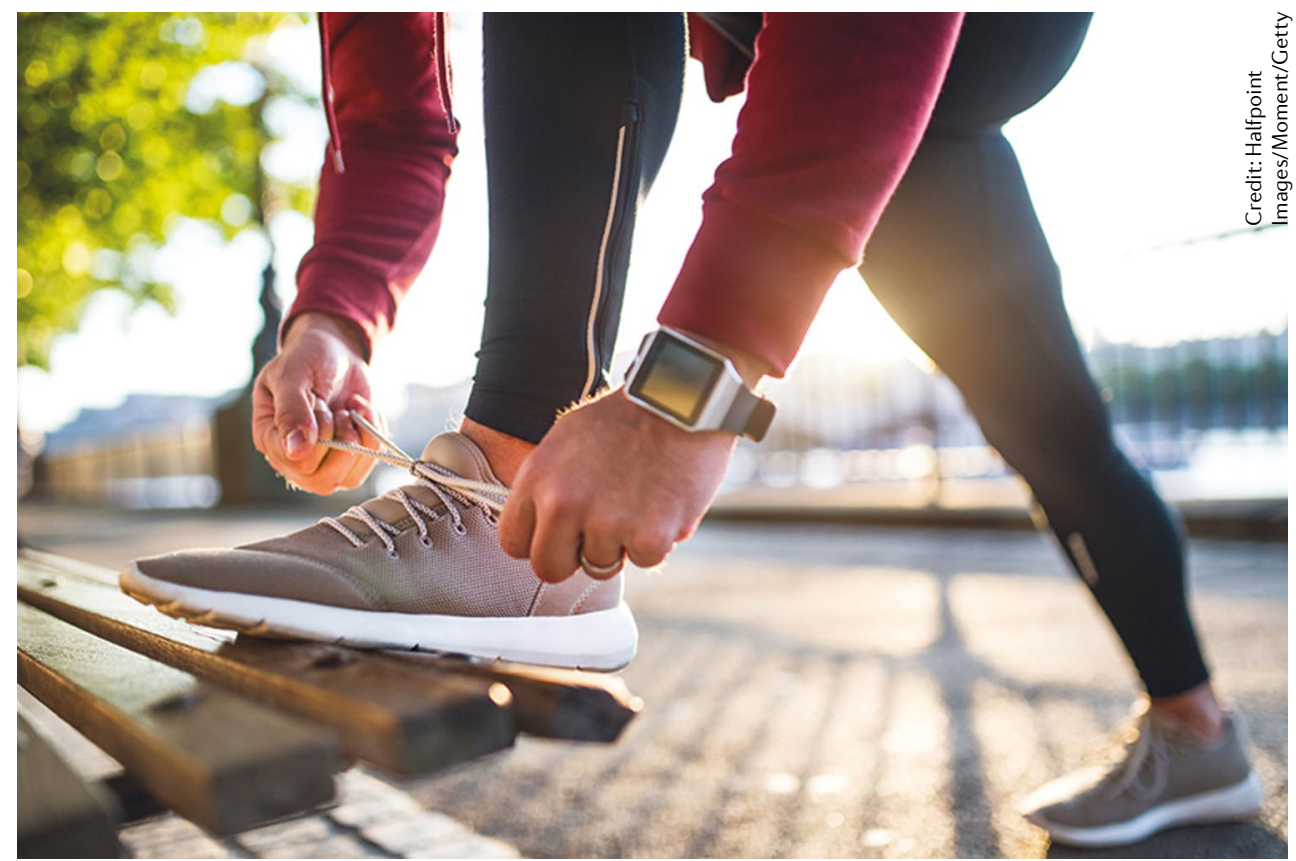

ALZHEIMER DISEASE

\section{An exercise-linked mediator of memory protection}

Several lines of evidence support a dysregulation of brain energy metabolism in patients with Alzheimer disease (AD). Interventions to treat metabolic disease including diabetes management and physical exercise have been shown to protect against $\mathrm{AD}$, but the underlying mechanisms are not fully understood. Now, a study in Nature Medicine reports that the exercise-induced myokine called irisin (and its precursor, FNDC5) is an important mediator of the benefit of exercise in $\mathrm{AD}$, suggesting a potential new avenue for therapeutic development.

Myokines are proteins secreted by muscle tissue that act as endocrine or paracrine signalling molecules. FNDC5/irisin is expressed in muscle in response to exercise and was known to stimulate expression of brain-derived neurotrophic factor in the hippocampus, suggesting a neuroprotective role in a brain region involved in learning and memory.

In the current study, Lourenco et al. began by measuring FNDC5/ irisin levels in the context of AD. They found reduced levels of the myokine in the hippocampi of latestage $\mathrm{AD}$ patients and in a mouse model of $\mathrm{AD}$ compared with agematched controls. Exposure of rat and human hippocampal brain slices to amyloid- $\beta(\mathrm{A} \beta)$ oligomers - soluble $\mathrm{A} \beta$ aggregates that accumulate in the $\mathrm{AD}$ brain - decreased FNDC5/irisin levels. Moreover, intracerebroventricular infusion of $\mathrm{A} \beta$ oligomers reduced hippocampal expression of FNDC5/irisin in wild-type mice.

Knockdown of FNDC5 through lentivirus-mediated delivery of short hairpin RNA into the brains of mice led to impaired maintenance of hippocampal long-term potentiation (LTP; the cellular correlate of memory formation) and of memory in a novel object recognition task. Conversely, boosting brain levels of FNDC5 in mice using an adenoviral vector protected against impaired performance in memory tests following intracerebroventricular infusion of $A \beta$ oligomers.

Quantitative PCR arrays of hippocampal gene expression in these mice suggested FNDC5/ irisin might protect against aberrant expression of synapse-related genes.

In an $\mathrm{AD}$ transgenic mouse model, adenoviral delivery of FNDC5 to the brain rescued performance in memory tests and restored LTP as measured in hippocampal slices.

To explore the mechanisms by which FNDC5/irisin confers neuroprotection in the setting of $\mathrm{AD}$, the team applied recombinant irisin to cultured mouse hippocampal neurons that had been exposed to $A \beta$ oligomers in vitro. Binding studies revealed no direct interaction between irisin and $A \beta$. However, irisin was found to stimulate a signalling pathway involved in memory formation (the cAMP-PKA-CREB pathway), which is known to be impaired in AD models.

To probe the possible link between the effects of FNDC5/ irisin and exercise, the authors subjected mice to a protocol of swimming for $1 \mathrm{~h}$ per day, 5 days per week for 5 weeks. This activity prevented hippocampal FNDC5/ irisin reductions and memory deficits in response to $A \beta$ oligomers. In $\mathrm{AD}$ transgenic mice, moreover, the exercise protocol abrogated impairments in LTP, and this protective effect was abolished by RNA knockdown of FNDC5.

Importantly, in $A \beta$-infused mice, peripheral administration of FNDC5 into the caudal vein prevented the reduction in hippocampal FNDC5/ irisin and memory impairments seen in mice that did not receive FNDC5. In addition, peripheral neutralization of FNDC5 with antibodies in $\mathrm{A} \beta$-infused mice and in $\mathrm{AD}$ transgenic mice abolished the protective effect of exercise on memory.

The authors note that further work is needed to identify the receptors for irisin in the brain. The potential ability of peripherally administered FNCD5/irisin to reach the brain and protect against synaptic dysfunction in the context of $\mathrm{A} \beta$-related pathology makes this metabolic mediator an attractive starting point for much needed therapeutic strategies in $\mathrm{AD}$.

Katie Kingwell

... memory impairments seen in mice that did not receive FNDC5
ORIGINAL ARTICLE Lourenco, M. V. et al. plasticity and memory defects in Alzheimer's models. Nat. Med. 25, 165-175 (2019) FURTHER READING Whitham, M. \& Febbraio, M. A. The ever-expanding myokinome: discovery challenges and therapeutic implications. Nat. Rev. Drug Discov. 15, 719-729 (2016) Exercise-linked FNDC5/irisin rescues synaptic 